\title{
Relato sobre a Utilização de Ferramenta com Realidade Aumentada no auxílio do Ensino-Aprendizagem de Topografia em Cursos Superiores
}

\author{
Laura Quevedo Jurgina ${ }^{1}$, João Inácio Moreira Bezerra ${ }^{1}$, Rafael Piccin Torchelsen ${ }^{1}$, \\ Leomar Soares da Rosa Júnior ${ }^{1}$ \\ ${ }^{1}$ Universidade Federal de Pelotas \\ \{lqjurgina, jimbezerra, Rafael.torchelsen, leomarjr\}@inf.ufpel.edu.br
}

Resumo. Este trabalho apresenta a avaliação de uma ferramenta educacional que utiliza Realidade Aumentada e Interface Tangível para auxiliar os alunos, de curso superior, na compreensão mais eficiente dos tópicos das disciplinas que abordam topografia e cartografia tridimensional. O trabalho foi realizado como uma ação do Grupo PET-Computação, tendo como público alvo alunos dos cursos de Computação e de Engenharia Hidrica da Universidade Federal de Pelotas. Os resultados alcançados demonstram a viabilidade de aplicação da ferramenta, a qual ainda é pouco explorada nas universidades brasileiras. Ainda, o estudo constata que a utilização de Realidade Aumentada e Interface Tangível é bem aceita pela comunidade acadêmica, podendo servir como tecnologia de auxílio aos tradicionais métodos de ensino-aprendizagem.

\begin{abstract}
This work presents an evaluation of an educational tool that uses Augmented Reality and Tangible Interface to allow undergraduate students to efficiently learn topography and $3 D$ cartography. The work was carried out by PET-Computação targeting students from Computer Science and Hydraulic Engineering courses at the Universidade Federal de Pelotas. The results show the tool feasibility, which is still barely explored in Brazilian universities. Furthermore, the study notes that the use of Augmented Reality and Tangible Interface is well accepted by the academic community and can serve as an aid technology to traditional teaching-learning methods.
\end{abstract}

\section{Introdução}

No contexto atual de ensino é possível notar o frequente uso de aulas expositivas pelos docentes em ambientes educacionais, todavia é necessário uso de metodologias que promovam um maior engajamento dos estudantes (Queiroz, Pinto e Silva 2019). A utilização de recursos computacionais precisa estar comprometida com métodos que possibilitem a concepção sobre o conhecimento. As ferramentas devem despertar a reflexão sobre o seu auto aprendizado, incentivando, desta maneira, a recepção solícita ao tema (Lopes, Silva e Almeida 2012).

Atualmente, existem diversas ferramentas computacionais aplicadas no Ensino Básico e no Ensino Superior, as aplicações podem ser encontradas facilmente na Web. Para 
o aprendizado de matemática no Ensino Médio, dentre vários softwares disponíveis, existe o emprego de MatLab perante - por exemplo - visualização de gráficos de funções (Vieira e Morais 2013). No Ensino Superior, ferramentas de auxílio a aprendizagem, tais como o SwicthCraft (Callegaro 2010) e o Karma (Klock, Ribas e Reis 2010), são utilizadas para exercitar os conceitos vistos por alunos de disciplinas de Técnicas e de Circuitos Digitais em cursos de Engenharia e de Computação. Outra modalidade que também vem crescendo é a adoção do uso de dispositivos móveis por alunos do Ensino Médio, onde existem aplicativos para acesso de questões preparatórias para vestibulares (EI\&T 2019) (Prates 2019). Da mesma forma, no Ensino Superior, aplicativos para smartphones vêm sendo adotados em disciplinas que exploram o uso de tecnologias em suas grades curriculares. O Circuit Scramble Suborbital (2019) e o Pythagorea Horis (2019) são exemplos de ferramentas utilizadas para este fim.

Recursos computacionais como Realidade Aumentada (RA) e Interfaces Tangíveis (TUI) têm sido empregadas em diversas ferramentas eletrônicas e áreas do conhecimento. Isso se deve ao fato destes recursos permitirem que o usuário interaja de maneira imersiva no problema a ser estudado ou resolvido (De Lima 2017) (Dos Reis e Gonçalves 2016).

A RA configura-se como uma modalidade de interface computacional avançada que busca alcançar a interação humano computador de uma forma mais natural e que mistura, em tempo real, objetos virtuais gerados por computador com elementos do ambiente físico. Os objetos virtuais são visualizados por meio da utilização de dispositivos tecnológicos de saída de dados, tais como óculos especiais (head mounted displays), smartphones e projetores, produzindo um ambiente único com impressão de continuidade ao usuário (Colpani e Homem 2016).

Segundo (De Lima 2017), temos um ambiente de realidade aumentada quando filmamos um local, em tempo real, e inserimos objetos virtuais, onde as cenas formadas dão a impressão de que os objetos virtuais pertencem ao mundo real. (Da Silva, Oliveira e Fernandes 2018) discutem a utilização de Realidade aumentada no ensino de Arquitetura de Computadores, onde a tecnologia é utilizada para integrar o material didático e as ferramentas de apoio ao aprendizado.

Com o avanço das tecnologias computacionais, especialmente àquelas relacionadas com a interação do usuário e o sistema digital, um novo campo de estudos se desenvolveu na computação, dando início às Interfaces Tangíveis. As TUI podem ser definidas como interfaces que compreendem interações realizadas em artefatos físicos, como estímulos para interferir no contexto e representações de informação digital. Simuladores veiculares são um exemplo de aplicação de Interfaces Tangíveis, uma vez que envolvem o uso de objetos físicos para manipulação de cenários virtuais, (Dos Reis e Gonçalves 2016).

Existem trabalhos que debatem sobre a inserção Realidade Aumentada no contexto de ensino para auxílio do aprendizado. (Wu 2013) e (El Sayed 2011) discutem sobre métodos para o sucesso da tecnologia como instrumento pedagógico. (Dos Reis 2016) discute, a partir de revisões literárias, sobre o conceito e a usabilidade de interfaces tangíveis. (Gluz et. Al. 2018) relata que um ambiente de ensino virtual tangível integra as tecnologias de realidade virtual e interfaces tangíveis. Assim, são conhecidas vantagens educacionais do uso de tais dispositivos em espaços educativos. Então, o foco de atenção 
centra-se em aprendizagens mais complexas que envolvem os conceitos científicos a partir de atividades mediadas.

Neste contexto, este trabalho possui o objetivo de avaliar uma ferramenta educacional desenvolvida para estudos topográficos, investigando, assim, a eficácia do seu emprego no aprendizado. Esta ferramenta faz uso de Realidade Aumentada e de Interface Tangível para permitir que os educandos consigam compreender de forma mais eficiente os conteúdos ensinados nas disciplinas que abordam topografia e cartografia tridimensional. A seção 2 deste trabalho apresenta a metodologia adotada. Na seção 3 os resultados obtidos são apresentados. Por fim, a seção 4 apresenta a discussão final e as conclusões.

\section{Metodologia}

O ensino de topografia inclui a cartografia e a representação tridimensional de relevos. Seu estudo está presente no ensino técnico e superior. Todavia seu estudo é complexo pela dificuldade enfrentada pelos educandos em converter a imagem em três dimensões em um mapa com duas dimensões, A Figura 1 ilustra a transformação.

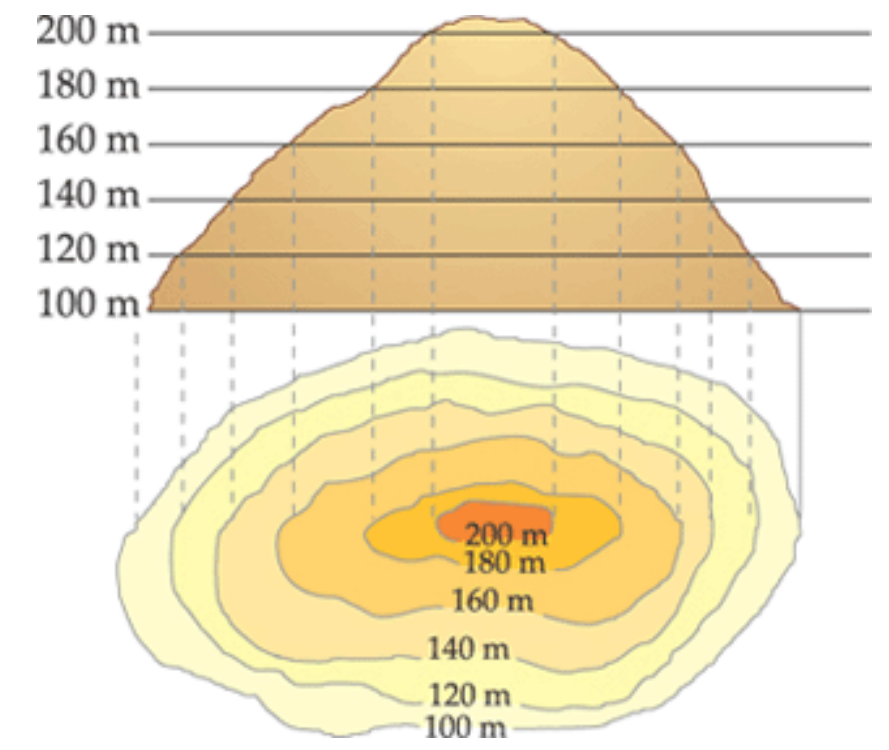

Figura 1. Mapa Topográfico e sua relação com o relevo a ser representado.

A ARSandBox consiste em uma aplicação que integra Realidade Aumentada e Interface Tangível para visualização 3D de ambiente geográfico, através de mapa topográfico (Darley 2017). A disposição da ARSandBox inclui uma caixa de areia com área de $1,5 \mathrm{~m}^{2}$; sensor Kinect 3D e projetor, localizados a $1 \mathrm{~m}$ de altura; um computador equipado com qualquer sistema operacional Linux, $2 \mathrm{~Gb}$ de memória RAM e placa gráfica Nvidia para executar o software que realiza a decodificação da área de interação (UCDAVIS 2019).

A Figura 2 apresenta o esquemático do sistema da ARSandBox. Seu funcionamento acontece por meio da manipulação da areia (TUI), recebendo a modificação estrutural dos usuários para formar relevos. $\mathrm{O}$ sistema capta a modificação física realizada e interpreta as novas curvas de níveis formadas, projetando as cores adequadas na areia. A Figura 3 ilustra a utilização da ARSandBox. 


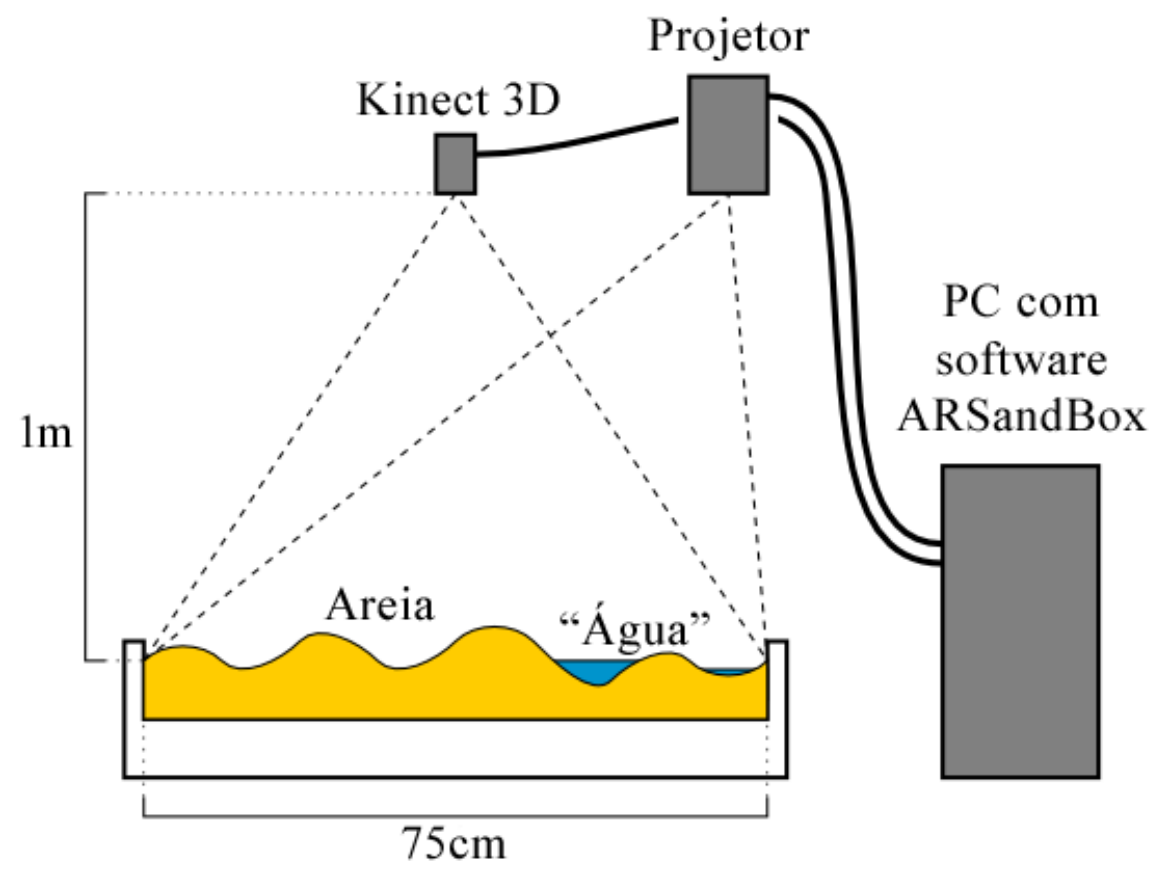

Figura 2. Arranjo típico do sistema da ARSandBox (UCDAVIS 2019).

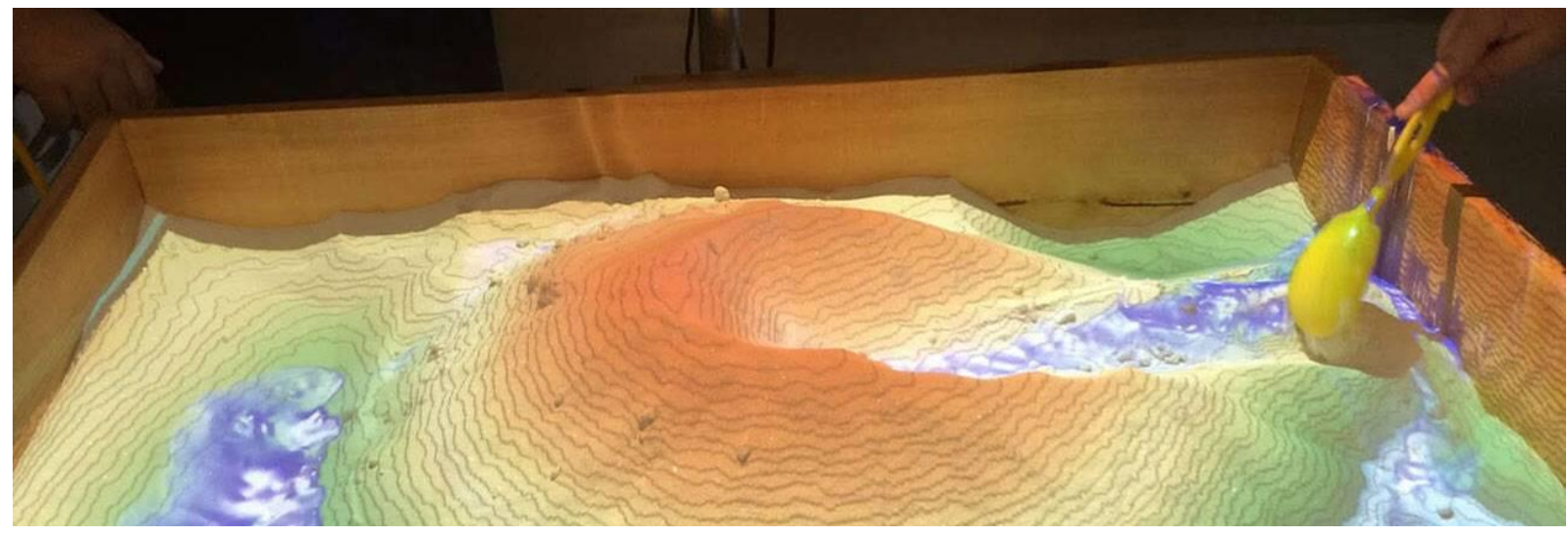

Figura 3. ARSandBox sendo utilizada para formar relevos.

Este projeto foi implantado em um laboratório da Universidade, através de uma iniciativa conjunta dos cursos de Ciência da Computação e de Engenharia Hídrica. Segundo (Darley 2017), a grande dificuldade em ensinar topografia para os alunos é a limitação que a superfície plana tem de representar os relevos de um terreno. Em uma folha de papel ou em um quadro só é possível desenhar em duas dimensões. A aplicação da ARSandBox contorna esta limitação, oferecendo a noção de um modelo tridimensional que aprimora o entendimento do aluno. O mais interessante é que ela consiste em uma TUI, onde o aluno pode moldar a superfície, permitindo novas descobertas (Darley 2017). A ARSandBox foi disponibilizada aos alunos que cursavam a disciplina de topografia. Estes, então, foram convidados a experimentar seu funcionamento.

A interação do público com a caixa fora inicialmente livre, com movimentos e contatos espontâneos para que os alunos conhecessem o ambiente. Após o período de 
reconhecimento algumas ações foram estimuladas e guiadas pelo pesquisador, tais como simular chuva e construir relevos de tamanhos diversos com o objetivo de demonstrar as funcionalidades da aplicação. Um mês após a experiência, um questionário eletrônico de avaliação foi fornecido aos usuários para coletar suas percepções relativas ao uso da ferramenta e impacto sentido na aprendizagem, de maneira a validar sua eficácia em cumprir o objetivo de auxílio.

\section{Avaliação}

Segundo (Carvalho 2005) quando os usuários possuem interação livre com a ferramenta, com movimentos e contatos espontâneos, para que os alunos conheçam ambiente, eles se sentem mais motivados a explorar a plataforma. Então, antes do contato do público com a caixa, houve um pequeno tutorial com informações prévias de funcionamento da estrutura e um período interação livre. Após o período de reconhecimento algumas ações foram estimuladas e guiadas pelo pesquisador, tais como simular chuva e construir relevos de tamanhos diversos com o objetivo de demonstrar as funcionalidades da aplicação. Além disso, foi proposto a construção de um relevo a partir do seu mapa em 2D para uma experiência completa.

A dinâmica teve duração de 50 minutos e participaram 83 alunos. Após a experiência, um questionário de avaliação foi fornecido aos usuários para coletar suas percepções relativas ao uso da ferramenta e impacto sentido na aprendizagem, de maneira a validar sua eficácia em cumprir o objetivo de auxílio no ensino de topografia.

A primeira pergunta buscou conhecer um pouco sobre o histórico dos alunos na disciplina de Topografia, questionando sobre suas reprovações, questionando quantas vezes o aluno cursou aquela disciplina. $\mathrm{O}$ resultado pode ser acompanhado na Figura 4:

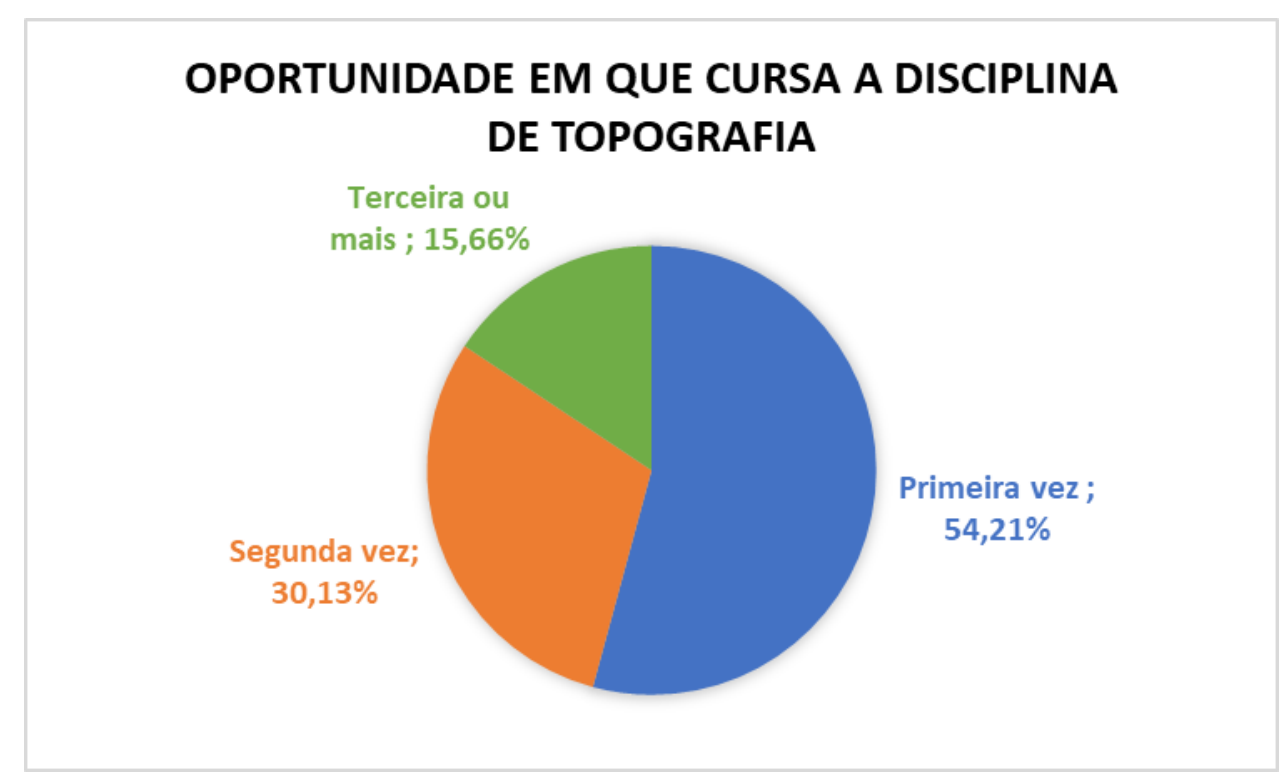

Figura 4. Gráfico com as porcentagens de avaliação da ARSandbox 
Considerando a interatividade do público com a ARSandBox, as seguintes perguntas foram realizadas, buscando identificar o impacto da ferramenta no processo de aprendizagem a partir da facilidade da utilização da ferramenta, pois precisa ser intuitiva e de simples compreensão. A eficiência é desejada para que o aluno tenha uma experiência fluida e que atenda aos seus objetivos. A aplicação também precisa auxiliar o usuário a prever seus erros na representação em duas dimensões, ou seja, com a manipulação da interface tangível é possível que detalhes do mapa sejam visualizados e manuseados, auxiliando no processo de cognição.

A avaliação também buscou verificar a adequação pedagógica da ferramenta, se ela possui correspondência entre suas aplicações e os conteúdos da disciplina de topografia. $\mathrm{O}$ critério de satisfação busca abordar se a ferramenta é capaz de atender o usuário no processo de aprendizagem. E por fim, a utilidade tem como objetivo saber se as ações propostas pela ARSandbox são suficientes para a experiência do aluno. A relação de perguntas está na Tabela 1 e as respostas estão na Figura 5.

Tabela 1. Relação dos critérios e perguntas realizadas

\begin{tabular}{|c|c|c|}
\hline $\begin{array}{l}\text { [1] Facilidade } \\
\text { Aprendizagem }\end{array}$ & na & "A interação foi realizada com facilidade?" \\
\hline [2] Eficiência & & "A ferramenta permite interação eficiente?" \\
\hline [3] Prevenção de erros & & $\begin{array}{l}\text { "Você consegue evitar erros na representação } \\
\text { cartográfica após utilizar a ferramenta?" }\end{array}$ \\
\hline [4] Adequação Pedagógica & & $\begin{array}{l}\text { "A ferramenta apresenta correspondência entre os } \\
\text { conteúdos da disciplina e suas aplicações?" }\end{array}$ \\
\hline [5] Satisfação & & "A ferramenta atende suas necessidades?" \\
\hline [6] Utilidade & & $\begin{array}{l}\text { "A ferramenta oferta as ações necessárias para sua } \\
\text { utilização?" }\end{array}$ \\
\hline
\end{tabular}

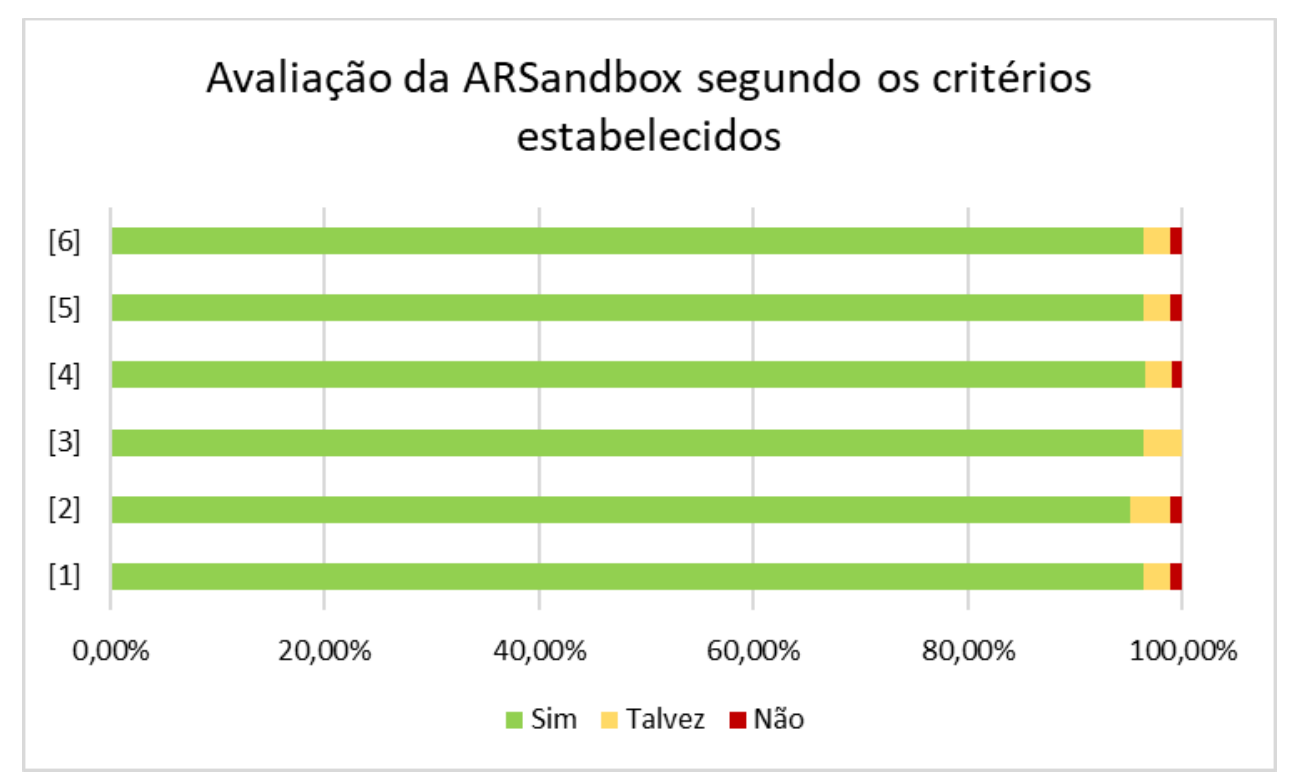

Figura 5. Gráfico com as porcentagens de avaliação da ARSandbox 
Segundo (Freire 2002) a capacidade de aprender implica na habilidade de aprender a substantividade do objeto aprendido e que a memorização mecânica do perfil do objeto não é aprendizado verdadeiro. A transformação de um mapa 3D em um plano pode ser algo complexo para aqueles que se sentem incapazes de visualizar tal ação. A partir da manipulação da areia e alteração da projeção, em tempo real, permitem e estimulam as percepções da convergência dos dois conceitos.

Os alunos também foram questionados sobre o impacto direto da ferramenta na sua compreensão sobre mapas topográficos, respondendo a seguinte pergunta: "Utilizar a ARSandbox é um diferencial para sua compreensão acerca da cartografia estudada na disciplina de topografia?”. O resultado está na Figura 5.

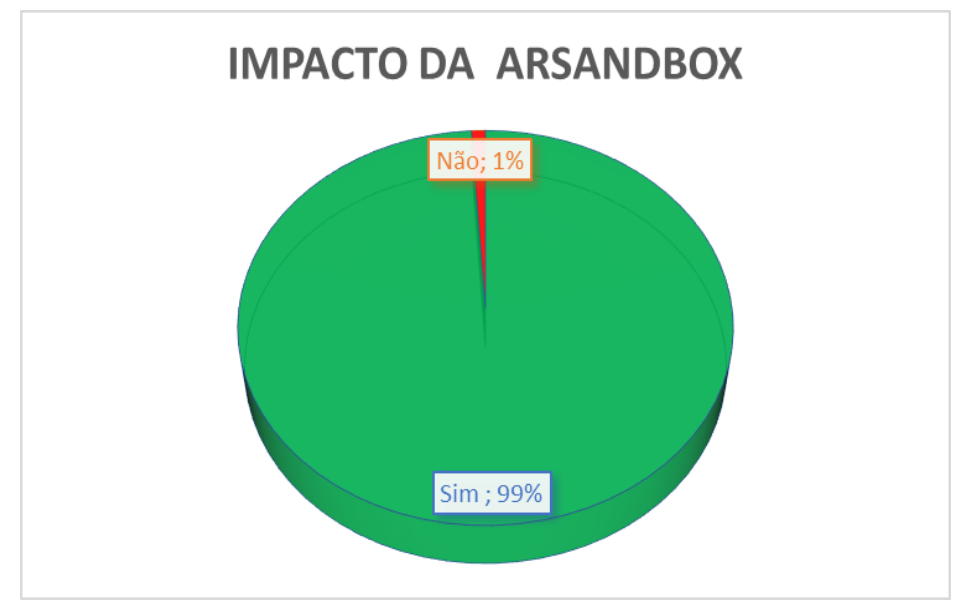

Figura 5. Gráfico com o impacto direto da ARSandbox

\section{Conclusões}

A partir das respostas positivas dos usuários à experiência com a ARSandBox, pode-se considerar a ferramenta um método diferencial no ensino de topografia. $\mathrm{O}$ uso combinado desta prática com o material 2D - já utilizado na teoria - faz com que ambas se complementem, atingindo todo o potencial de aprendizado.

Com o avanço da tecnologia, o emprego da mesma na simplificação de atividades torna-se inevitável. A RA não pode ser visualizada apenas como avanço utilizado em um mundo gamer, porém sim como ferramenta empregável diariamente. No meio educacional as vantagens oferecidas pela mescla de realidades integradas a uma interface tangível trazem uma colaboração visual e sensível na compreensão de projetos em $3 \mathrm{D}$ que são abstratos quando no plano.

Para melhor validação serão realizadas outras avaliações no futuro, separando os usuários em dois grupos diferentes, com a construção de um mapa 2D antes da experiência e outro mapa após. Porém um conjunto utilizará a caixa e outro não, para que depois sejam confrontados os resultados expondo os dois lados do processo ensino-aprendizagem: $\mathrm{O}$ impacto verificado pelo educador e a percepção do educando.

A avaliação presente neste trabalho mostra que a experiência com a ARSandbox é uma ferramenta de potencial no processo ensino-aprendizagem sob perspectiva do usuário. 
Assim como (Freire 2002) define que ensinar exige compreender que a educação é uma forma de intervenção no mundo, também devemos refletir sobre a importância e dever de aproximar e aplicar a tecnologia no ensino.

\section{Agradecimentos}

Este trabalho foi realizado com o apoio da Universidade Federal (UFPel), dos Cursos de Engenharia Hídrica, Ciência da computação e Engenharia de Computação do Centro de Desenvolvimento Tecnológico da UFPel, a Coordenação de Aperfeiçoamento de Pessoal de Nível Superior (CAPES), Conselho Nacional de Desenvolvimento Científico e Tecnológico (CNPq) e Fundação de Amparo à pesquisa do Estado do Rio Grande do Sul.

\section{Referências}

Callegaro, V., Marques, F., Klock, C., Da Rosa JR, L., Ribas, R. and REIS, A. (2010) SwitchCraft. In: 23rd Symposium on Integrated Circuits and System Design.

Campos, André; Gardiman, Renato; Madeira, Charles. Uma ferramenta gamificada de apoio à disciplina introdutória de programação. In: WORKSHOP SOBRE EDUCAÇÃO EM COMPUTAÇÃO (WEI), 23., 2015, Recife. Anais. Porto Alegre: Sociedade Brasileira de Computação, 2015. p. 356-365. ISSN 2595-6175

Carvalho, Ana A. A. (2005). Como olhar criticamente o software educativo multimídia.

Colpani, R. and Homem, M. (2016) Realidade Aumentada e Gamificação na Educação: uma aplicação para auxiliar no processo de aprendizagem de alunos com deficiência intelectual. In: Revista Brasileira de Informática na Educação.

Da Silva, Geofrangite Câmara; Oliveira, Leiva Casemiro; Fernandes, Sílvio Roberto. Uso de Realidade Aumentada para Ensino de Arquitetura de Computadores com MIPS. In: WORKSHOP SOBRE EDUCAÇÃO EM COMPUTAÇÃO (WEI), 26., 2018, Natal. Porto Alegre: Sociedade Brasileira de Computação, 2018. ISSN 2595-6175

Darley, N. et al. (2017) "Tangible Interfaces: An Analysis of User Experience Using the ARSandbox Project. In: Simpósio Brasileiro Sobre Fatores Humanos Em Sistemas Computacionais.

De Lima A., et al. (2017) A importância da realidade aumentada aplicada à educação. In: IV Congresso Nacional de Educação

Dos Reis, A. (2016) Interfaces Tangíveis em Simuladores Veiculares: Componentes para um Protocolo de Avaliação de Usabilidade. 129f. Dissertação (mestrado) - Programa de Pós-Graduação em Design e Expressão Gráfica, Universidade Federal de Santa Catarina.

Dos Reis, A. and Gonçalves, B. (2016) Interfaces Tangíveis: Conceituação e Avaliação. Estudos em Design, v.24, n.2, p.92-111.

EI\&T. AppProva ENEM 2018 - "Simulados e Questões Inéditas" https://itunes.apple.com/br/app/appprova-enem-2018/id639910973

El Sayed, N., Zayed, H. and Sharawy, M. (2011) Augmented reality student card. Computers \& Education. 
Freire, P. (2002), Pedagogia da autonomia, saberes necessários à prática educativa, 25 edição

Horis,

https://play.google.com/store/apps/details?id=com.hil_hk.pythagorea

"Pythagorea:

Jurgina, Laura Quevedo; Zanandrea, Régis; Rosa Júnior, Leomar Soares; Marques, Felipe de Souza. LogicFlow: Uma Ferramenta Para o Auxílio de Ensino-aprendizagem de Circuitos Digitais. In: SIMPÓSIO BRASILEIRO DE INFORMÁTICA NA EDUCAÇÃO, 31., 2020, Online. Anais. Porto Alegre: Sociedade Brasileira de Computação, 2020. p. 322-331.

Klock, C., Ribas, R. And Reis, A. "Karma: um ambiente para o aprendizado de síntese de funções Booleanas” Revista Brasileira de Informática na Educação, v.18, p.33-42, 2010.

Lopes, M., Silva R. and Almeida A. (2012) "A importância do uso das ferramentas computacionais no ensino da disciplina fenômenos de transportes nos cursos de engenharia”, Anais do Congresso Brasileiro de Educação em Engenharia. v.XL. p.103.

Padrão, M. (2016) "Entenda a realidade aumentada, recurso por trás do sucesso de Pokémon Go" https://tecnologia.uol.com.br/noticias/redacao/2016/08/05/entendaarealidade-aumentada-recurso-por-tras-do-sucesso-de-pokemon-go.htm

Prates, L. (2019) "Studos - Simulados ENEM e Vestibulares" Apple Store, 30 mar. 2019. Acessado em 30 mar. 2019. Online. Disponível em: https://itunes.apple.com/br/app/studos/id819499553

Queiroz, Rafael; Pinto, Fabrício; Silva, Paulo. IslandTest: jogo educativo para apoiar o processo ensino-aprendizagem de testes de software. In: WORKSHOP SOBRE EDUCAÇÃO EM COMPUTAÇÃO (WEI), 27., 2019, Belém. Anais. Porto Alegre: Sociedade Brasileira de Computação, 2019. p. 533-542. ISSN 2595-6175.

Raeder, Mateus; Py, Mônica; Rigo, Sandro; Pinheiro, Josaine. L2PM: relato de uma experiência sobre o ensino integrado de Lógica, Programação e Matemática para Computação. In: WORKSHOP SOBRE EDUCAÇÃO EM COMPUTAÇÃO (WEI), 24., 2016, Porto Alegre. Anais [...]. Porto Alegre: Sociedade Brasileira de Computação, 2016. p. 191-200

Suborbital, G. (2019) "Circuit Scramble https://play.google.com/store/apps/developer?id=Suborbital+Games

UCDAVIS. (2019) “Augmented Reality Sandbox”. UC Davis' W.M. Keck Center for Active Visualization in the Earth Sciences. https://arsandbox.ucdavis.edu/

Vieira, C. And Morais V. (2013) "MATLAB, Curso Completo. FCA."

$\mathrm{Wu}$, H.(2018). "Current status, opportunities and challenges of augmented reality in education”. Computers \& Education, Elsevier Ltd.

Gluz, J., Passerino, L., Preuss, E., Baierle, I. L. F. \& Cimadevila (2018). Ambiente Virtual Tangível de Ciências numa Perspectiva Inclusiva. In: Anais do SIMPÓSIO BRASILEIRO DE INFORMÁTICA NA EDUCAÇÃO, 29, 2018. Porto Alegre: Sociedade Brasileira de Computação, 2018. pp. 545-544. 